\title{
MODELO DE LA DISTRIBUCIÓN DE ESPECIES VECTORES DE LEISHMANIASIS DEL GÉNERO Lutzomyia (DIPTERA: PSYCHODIDAE) EN MÉRIDA VENEZUELA
}

\author{
MODELING THE DISTRIBUTION OF VECTOR SPECIES OF LEISHMANIASIS OF GENUS \\ Lutzomyia (DIPTERA: PSYCHODIDAE) IN MERIDA VENEZUELA
}

\author{
Maria Rujano, Luzmary Oraá, Yorfer Rondón, Mireya Sánchez, Maritza Rondón, Yetsenia Sánchez, Masyelly \\ Rojas, Nestor González, Dalmiro Cazorla y Elsa Nieves
}

\begin{abstract}
RESUMEN
Los análisis espaciales han impulsado el desarrollo de modelos que permiten predecir la distribución espacial de las especies. Con estas técnicas se estima la distribución geográfica potencial, con la representación de áreas de ocurrencia de las especies, a partir de la asociación entre los registros de presencia y las variables ambientales del sitio. El objetivo de este estudio fue evaluar la eficiencia de dos modelos, el GARP y el MaxEnt, para predecir la distribución potencial de las principales especies de Lutzomyia, en un área endémica de leishmaniasis en Venezuela. Se utilizaron 190 puntos georreferenciados en el Estado Mérida Venezuela con presencia de flebotominos. Estos puntos se dividen en 67 puntos con presencia de Lutzomyia youngi, 51 de Lutzomyia gomezi, 40 de Lutzomyia ovallesi y 32 de Lutzomyia walkeri. Las variables bioclimáticas fueron tomadas de la base de datos del Worldclim para la zona 23. Los resultados muestran que ambos modelos presentaron un buen desempeño y sus rangos de distribución son consistentes entre sí. Aunque el GARP predice una mayor distribución potencial para Lutzomyia youngi y Lutzomyia gomezi, el MaxEnt genera predicciones más ajustadas de probabilidades de ocurrencia, determinadas por las variables bioclimáticas que caracterizan la distribución potencial de cada especie. Se recomienda en mayor grado el uso del MaxEnt, porque presenta una proyección de la distribución más acorde a la contribución de las variables bioclimáticas ya que aporta datos importantes para entender la dinámica de distribución de las especies de Lutzomyia y el riesgo de transmisión de leishmaniasis en el estado Mérida, Venezuela, información relevante para los entes de control.
\end{abstract}

PALABRAS CLAVES: distribución de especies; flebotominos; GARP; MaxEnt; modelos

\begin{abstract}
Spatial analyzes have prompted the development of models to predict the spatial distribution of species. These techniques estimate the potential geographical distribution areas by representing the occurrence of the species from the association between the presence records and environmental variables of the site. The aim of this study was to evaluate the efficiency of two models, MaxEnt and GARP, to predict the potential distribution of the main species of Lutzomyia in an endemic area of leishmaniasis in Venezuela. We use 190 georeferenced points of sandflies captured in the state of Merida, discriminated in 67 points for Lutzomyia youngi, 51 for Lutzomyia gomezi, 40 for Lutzomyia ovallesi and 32 for Lutzomyia walkeri. We take the climatic variables from the database Worldclim for area 23. The results show that both models had a good performance and their distribution ranges are consistent between themselves. Although GARP predicts a greater potential distribution for Lutzomyia youngi and Lutzomyia gomezi, MaxEnt generates more accurate predictions of probabilities of occurrence, determined by the bioclimatic variables that characterize the potential distribution of each species. It is considered better use MaxEnt because it presents a projection of the distribution according to contribution of bioclimatic variables as it provides important information for understanding the dynamics of distribution of Lutzomyia species and the risk of transmission of leishmaniasis in the state Merida-Venezuela, information relevant to the control entities.
\end{abstract}

KEY WORDS: distribution of species; sandflies; GARP; MaxEnt; models

Laboratorio de Parasitología Experimental (LAPEX), Departamento de Biología, Facultad de Ciencias y Laboratorio de Sostenibilidad y Ecodiseño, Facultad de Ciencias Forestales y Ambientales, Facultad de Arquitectura y Diseño, Universidad de Los Andes, 5101 Mérida, Venezuela. (M.R). Laboratorio de Parasitología Experimental (LAPEX), Departamento de Biología, Facultad de Ciencias, Universidad de Los Andes, 5101 Mérida, Venezuela (L.O), (Y.R), (M.S), (M.R), (Y.S), (M.R), (N.G), (E.N). Laboratorio de Entomología, Parasitología y Medicina Tropical (LEPAMET), Universidad Nacional Experimental Francisco de Miranda, 4101 Falcón, Venezuela (D.C). Autor de correspondencia: nievesbelsa@gmail.com. 


\section{INTRODUCCIÓN}

Las especies del género Lutzomyia (Diptera: Psychodidae), son vectores implicados en la transmisión de la leishmaniasis, enfermedad que representa un problema de salud pública con serias implicaciones para la sociedad (Stockdale y Newton, 2013; OMS, 2015). La dinámica de transmisión del parásito Leishmania, causante de la leishmaniasis, es determinada por factores propios del parásito, del hospedador vertebrado y de la población de flebotominos transmisores (Githeko et al., 2009). La deforestación, la incursión en las explotaciones agrícolas, los asentamientos humanos en zonas boscosas y la presencia de animales silvestres y/o domésticos, son las principales causas que inciden en la ocurrencia de estos vectores, con características epidemiológicas particulares en cada región (Feliciangeli, 1988; De Lima et al., 2006; Rodríguez et al., 2007; Nieves et al., 2008). En los últimos años, producto del cambio climático y de modificaciones ambientales, ha aumentado el contacto vector-hospedador, lo que ocasiona un expansión de la leishmaniasis hacia nuevas áreas (Valderrama et al., 2011).

La adaptación espacio-temporal de las especies es determinante en la interacción vector-hospedador, lo cual depende principalmente de factores de movilidad del insecto (De Lima et al., 2010). Cada especie de flebotominos ocupa un nicho ecológico particular, que depende del banco genético de la población y de las características conductuales específicas, lo que permite que algunas especies se adapten a nuevos ambientes con distintos grados de perturbación ecológica y potencialmente se incremente el riesgo de transmisión en diferentes áreas (Githeko et al., 2009; Nieves et al., 2014a). La distribución geográfica de los flebotominos está correlacionada con factores ecológicos, climáticos y antropogénicos, que han evolucionado a lo largo de los años en un ciclo propio en cada región, esto ha causado un impacto directo sobre la abundancia del vector y la transmisión de leishmaniasis (Valderrama et al., 2011). Es necesario entender la dinámica de distribución de los flebotominos vectores, para poder comprender la complejidad epidemiológica en la transmisión de la leishmaniasis, aspecto a considerar al momento de establecer políticas de prevención (Alvar et al., 2012).

Diferentes modelos predictivos se han desarrollado para describir la distribución potencial de las poblaciones (Anderson et al., 2003; Carnaval y Moritz, 2008; Pliscoff y Fuentes, 2011; Valera et al., 2014), ligados al avance de los Sistemas de Información Geográfica y a técnicas estadísticas (Mateo et al., 2011). Muchos modelos predicen la presencia probable de una especie en áreas no exploradas, basados en los sitios de presencia, correlacionados con variables bioclimáticas (Chapman y Busby, 1994; Anderson et al., 2003; Foley et al., 2010; Fischer et al., 2011; Donalisio et al., 2012). Estos modelos son propuestos para entender la distribución geográfica de las especies y ofrecer un mapa de distribución, lo que permite comprender la relación entre diferentes variables y dicha distribución (Pliscoff y Fuentes, 2011).

Algunos modelos utilizan técnicas geoestadísticas con relación a variables climáticas, se generan perfiles bioclimáticos de las especies transmisoras, que son fundamentales en las políticas de control en zonas particulares (Fischer et al., 2011). Entre los métodos de modelización, están los que se basan en técnicas descriptivas que requieren datos de presencia, entre ellos BIOCLIM, DOMAIN y BIOMAP (Pliscoff y Fuentes, 2011). Otros se basan en técnicas discriminantes, que requieren datos de presencias y generan sus propias pseudoausencias; entre ellos tenemos los Árboles de Clasificación y Regresión Logística, Modelos Lineales Generalizados, Redes Neuronales, Modelos Aditivos Generalizados y el MaxEnt. También existen modelos que combinan técnicas descriptivas y discriminantes, generan sus propias ausencias; entre estos el más utilizado es el GARP y los modelos de consenso como el BIOMOD (Tognelli et al., 2009; Wang et al., 2010; Mateo et al., 2011; Pliscoff y Fuentes, 2011; Chen y Lei, 2012; Vessella y Schirone, 2013). Los modelos con GARP (Stockwell y Noble, 1992) y MaxEnt (Phillips et al., 2006), se encuentran entre los algoritmos más utilizados en la literatura, a pesar de su nivel de complejidad (Carnaval y Moritz, 2008; Torres y Jayat, 2010; Mateo et al., 2011; Rubio y Acosta, 2011; Romo et al., 2012; Acosta y Vergara, 2013; Valera et al., 2014). Los estudios muestran que el desempeño de los modelos de distribución, puede variar dependiendo de la especie que se trabaje, el área de estudio y el número de localidades de presencia utilizadas. Por lo cual se hace necesario evaluar cuál es la mejor técnica de modelación en cada situación concreta (Elith et al., 2006; Hernández et al., 2006; Pearson et al., 2006; González et al., 2011; Pliscoff y Fuentes, 2011).

Varios modelos han sido aplicados a poblaciones de flebotominos en diferentes contextos y aportes 
(Traviezo, 2007; González et al., 2011; Abdel et al., 2012; Donalisio et al., 2012; Quintana et al., 2013). Thomson et al. (1999), utilizaron Regresión Logística y referencian a Phlebotomus orientalis en diferentes habitas en un mapa de riesgo de leishmaniasis visceral, determinaron que la media máxima anual diaria de temperatura y el tipo de suelo son las determinantes ecológicas de la distribución de este transmisor. Sánchez et al. (2015), aplicaron MaxEnt y determinaron la distribución potencial de las principales especies de flebotominos en Venezuela. A pesar de la alta endemicidad de la leishmaniasis en el Estado Mérida en Venezuela y de la importancia biológica y de salud pública de los flebotominos, no existe un estudio local que muestre la distribución espacial de las principales especies transmisoras. En el presente trabajo se evalúa la distribución potencial de las principales especies de flebotominos, vectores de leishmaniasis, en el Estado Mérida, Venezuela, con la utilización de dos modelos, el GARP y el MaxEnt, con el fin de aportar datos relevantes sobre la modelación de flebotominos vectores de Leishmania, para comprender la epidemiologia de la leishmaniasis necesarios para establecer nuevas políticas de prevención en el estado.

\section{METODOLOGÍA}

\section{Área de Estudio}

El Estado Mérida se localiza al occidente de Venezuela, entre los $07^{\circ} 34^{\prime} 60,0^{\prime \prime}$ y $09^{\circ} 21^{\prime} 0,0^{\prime \prime}$ ' de latitud Norte y los $70^{\circ} 30^{\prime} 0.0^{\prime \prime}$ y $72^{\circ} 0^{\prime} 0.0^{\prime \prime}$ de Longitud Oeste (Figura 1). Debido a las variaciones altitudinales que presenta, tiene una variación climática que va desde los climas semiáridos en las partes más bajas, con precipitaciones que oscilan entre 520 y $1800 \mathrm{~mm}$ y temperaturas medias anuales cercanas a $27{ }^{\circ} \mathrm{C}$, hasta los climas de páramo, localizados por encima de los $2500 \mathrm{~m}$ de altitud, con precipitaciones anuales cercanas a 1400 mm y temperaturas que oscilan entre $18{ }^{\circ} \mathrm{C}$ a $12{ }^{\circ} \mathrm{C}$ y en las zonas de mayor altura alcanzan los $0{ }^{\circ} \mathrm{C}$. Las múltiples combinaciones de factores tales como altitud, temperatura y precipitación, condicionan la existencia de una gran variedad de tipos de vegetación que varían entre arbustivas y de sabana en zonas bajas, pasando por formaciones de selva nublada en las zonas más altas, hasta llegar a una vegetación de páramo en las zonas de mayor altitud (Ataroff y Sarmiento, 2004).

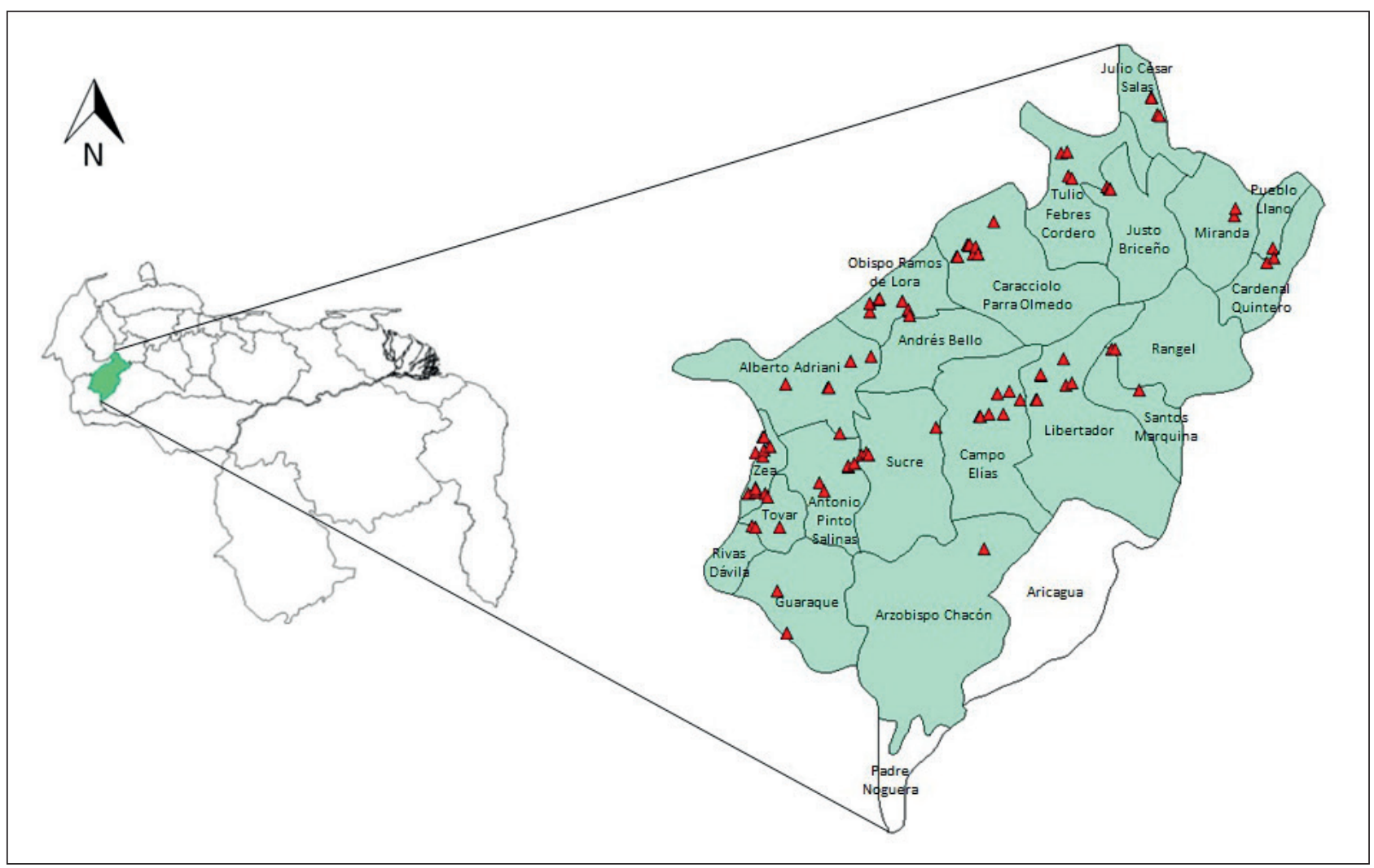

Figura 1. Ubicación geográfica del estado Mérida y los puntos de captura de flebotominos en 21 de los 23 municipios del estado Mérida, Venezuela. 


\section{Datos de presencia}

Las capturas de flebotominos se realizaron en 21 de los 23 municipios del Estado Mérida, se abarcó un gradiente altitudinal entre 61 y $2099 \mathrm{~m}$. Para la captura de los flebotominos se emplearon trampas de Shannon, trampas adhesivas, trampas CDC y aspiración directa, como lo describe Nieves et al. (2014a). La identificación de los ejemplares se hizo mediante morfología externa e interna, según los criterios expuestos por Young y Duncan (1994). Todos los puntos de captura fueron georreferenciados mediante un equipo GPS-Garmin-Oregón 550 (UTM REGVEN WGS-84). Se recolectaron 3400 flebotominos, de los cuales $2469(72,41 \%)$ fueron de las cuatro especies más abundantes (L. youngi, L. gomezi, L. ovallesi y L. walkeri), encontradas en 79 localidades del área de estudio. La Tabla 1 muestra la abundancia de las especies obtenidas por municipio en el Estado Mérida.

Para el análisis de la distribución espacial de las especies mediante el GARP y el MaxEnt, se emplearon 190 puntos georreferenciados de únicas ocurrencias de las especies más abundantes, discriminados en 67 registros de L. youngi, 51 de L. gomezi, 40 de $L$. ovallesi y 32 de L. walkeri. La cantidad de registros por especie utilizados se encuentraban por encima del valor mínimo recomendado (30) para obtener modelos estadísticamente válidos (Wiszet al., 2008).

Tabla 1. Abundancia de las principales especies del género Lutzomyia por municipios en el Estado Mérida.

\begin{tabular}{lrrrrr}
\hline Municipios & L. youngi & L. gomezi & L. ovallesi & L. walkeri & Total \\
\hline Alberto Adriani & 1 & 23 & 4 & 0 & 28 \\
Andrés Bello & 84 & 9 & 13 & 7 & 113 \\
Antonio Pinto Salinas & 15 & 0 & 5 & 21 & 41 \\
Arzobispo Chacón & 0 & 54 & 0 & 0 & 54 \\
Campo Elías & 250 & 0 & 16 & 0 & 266 \\
Caracciolo Parra Olmedo & 3 & 4 & 0 & 16 & 23 \\
Cardenal Quintero & 0 & 0 & 1 & 0 & 1 \\
Guaraque & 24 & 22 & 0 & 1 & 47 \\
Julio Cesar Salas & 4 & 238 & 0 & 0 & 242 \\
Justo Briceño & 311 & 0 & 0 & 0 & 311 \\
Libertador & 12 & 0 & 0 & 0 & 12 \\
Miranda & 10 & 0 & 0 & 0 & 10 \\
Obispo Ramos De Lora & 0 & 101 & 1 & 3 & 105 \\
Pueblo Llano & 0 & 0 & 0 & 0 & 0 \\
Rangel & 0 & 0 & 0 & 0 & 0 \\
Rivas Dávila & 21 & 0 & 0 & 0 & 21 \\
Santos Marquina & 1 & 0 & 0 & 0 & 1 \\
Sucre & 210 & 0 & 42 & 0 & 252 \\
Tovar & 46 & 0 & 0 & 0 & 46 \\
Tulio Febres Cordero & 0 & 12 & 0 & 0 & 12 \\
Zea & $\mathbf{1 2 1 3}$ & $\mathbf{7 8 3}$ & $\mathbf{3 3 8}$ & $\mathbf{1 3 5}$ & $\mathbf{2 4 6 9}$ \\
\hline Total & & & & 254 \\
\hline
\end{tabular}




\section{Datos climáticos}

Para la construcción de los modelos predictivos con GARP y MaxEnt, se utilizaron 19 variables bioclimáticas, que corresponden a capas ambientales en formato raster para la zona 23, con una resolución de 30 arco segundos (1 km) (Hijmans et al., 2005a), derivadas de la interpolación de datos medios mensuales de precipitación y temperatura registrados en estaciones meteorológicas durante el período 1950-2000. Estos datos fueron tomados de la base de datos Worldclim (http://www.worldclim.org). Las variables utilizadas fueron las siguientes: $\mathrm{BIO1}$, temperatura promedio anual $\left({ }^{\circ} \mathrm{C}\right)$; BIO2, rango diurno de temperatura $\left({ }^{\circ} \mathrm{C}\right)$; BIO3, isotermalidad $\left({ }^{\circ} \mathrm{C}\right)$; $\mathrm{BIO} 4$, estacionalidad de temperatura (\%); BIO5, temperatura máxima del período más caliente $\left({ }^{\circ} \mathrm{C}\right)$; BIO6, temperatura mínima del período más frío $\left({ }^{\circ} \mathrm{C}\right)$; BIO7, rango anual de temperatura $\left({ }^{\circ} \mathrm{C}\right)$; $\mathrm{BIO8}$, temperatura media en el trimestre más lluvioso $\left({ }^{\circ} \mathrm{C}\right)$; BIO9, temperatura promedio en el trimestre más seco $\left({ }^{\circ} \mathrm{C}\right)$; BIO10, temperatura promedio en el trimestre más caluroso $\left({ }^{\circ} \mathrm{C}\right)$; BIO11, temperatura promedio en el trimestre más frío $\left({ }^{\circ} \mathrm{C}\right)$; BIO12, precipitación anual (mm); BIO13, precipitación en el período más lluvioso (mm); BIO14, precipitación en el período más seco (mm); BIO15, estacionalidad de la precipitación (\%); BIO16, precipitación en el trimestre más lluvioso $(\mathrm{mm})$; BIO17, precipitación en el trimestre más seco (mm); BIO18, precipitación en el trimestre más caluroso $(\mathrm{mm})$; y BI019, precipitación en el trimestre más frío (mm). En este estudio no se pudieron incluir las variables topográficas y ecológicas, ya que para el momento del estudio no se contaron con capas de datos de estas variables con una buena resolución para el Estado Mérida.

\section{Modelos de distribución potencial de flebotominos}

Para predecir y analizar la distribución potencial de las principales especies de Lutzomyia en el Estado Mérida, se usaron los algoritmos GARP y MaxEnt. Se modeló el nicho fundamental, que corresponde a las zonas potencialmente idóneas de las especies, determinadas mediante la relación entre los puntos de presencia conocidos y las variables medio ambientales del sitio. Ambos métodos utilizan como entrada un conjunto de localidades donde se conoce que la especie está presente y un grupo de coberturas geográficas que representan los parámetros ambientales que pueden limitar la capacidad de supervivencia de la especie. En los dos modelos, las no presencias son simuladas como pseudoausencias, datos que son tomados al azar de la región de interés (Extent geográfico), para caracterizar el ambiente en general (Elith et al., 2006; Mateo et al., 2011; Valera et al., 2014).

Los modelos de distribución potencial de cada una de las especies, obtenidos con ambos métodos, fueron sumados algebraicamente para una mejor visualización de los modelos predictivos, para el procesamiento de la información se utilizó el programa Diva GIS v7.5 (http://www.diva-gis.org/, versión 7.5, Hijmans et al., 2005b). De esta forma se obtuvo un mapa final de idoneidad del hábitat, con un rango de valores continuos entre 0 y 10 por cada especie (Anderson et al., 2003; Wang et al., 2010). El rango de 0-2 (color blanco), indica las áreas donde los modelos coinciden en la ausencia de la especie; el rango 2-4 (color verde), señala las áreas con baja probabilidad de presencia; de 4 a 6 (color amarillo), se tienen las áreas con probabilidad media de presencia; de 6-8 (color naranja), las áreas con alta probabilidad de presencia y de 8-10 (color rojo), las áreas en donde ambos modelos predicen que las condiciones climáticas son excelentes para la presencia de las especies (Anderson et al., 2003).

\section{GARP}

El algoritmo GARP (Genetic algorithm for rule-set production; Stockwell y Noble, 1992), está disponible en el programa Desktop Garp v1.1.6 (Scachetti, 2002). El GARP procura encontrar de manera iterativa las correlaciones entre la presencia y la ausencia de las especies, con los valores de los parámetros ambientales, para lo cual se emplean diferentes tipos de reglas: atómica, envoltura climática, negación de la envoltura climática y regresión logística. En cada corrida se genera y se evalúa un modelo binario de distribución (presencia $=1$, ausencia $=0$; Scachetti, 2002) .

Para cada tipo de regla se implementa un método diferente, definido por (1) Las variables ambientales que caracterizan cada uno de los puntos de presencia de la especie (Atómicas); (2) El rango de valores que cada variable ambiental abarca, lo que define la tolerancia de la especie al medio (Envoltura climática); (3) El rango de valores de las variables que pueden ser irrelevantes para la especie (Negación de la envoltura climática) y (4) El resultado es transformado en un valor de probabilidad. Si la probabilidad se encuentra por encima del valor establecido (0-1), entonces el modelo asume que la presencia de la especie es posible (Regresión logística; 
Stockwell y Peters, 1999). Se generaron 10 modelos por cada especie con un límite de convergencia de 0,01 y un número máximo de 100 iteraciones. Así mismo, se emplearon los cuatro tipos de reglas (Anderson et al., 2003; Peterson et al., 2007; Wang et al., 2010; Quintana y Salomón, 2011) y se utilizó un umbral de omisión suave, con un $10 \%$ de error de omisión y un 50 \% de error de comisión en la distribución (valores por defecto) (Peterson et al., 2007).

\section{MaxEnt}

El algoritmo MaxEnt (Maximum Entropy; Phillips et al., 2006), basado en el análisis de máxima entropía, está disponible en el programa MaxEnt v3.3 (Phillips y Dudik, 2008). MaxEnt estima la probabilidad continua (en términos de ganancia), de ocurrencia de la especie, buscando la distribución de máxima entropía (lo más uniforme posible), sujeta a la condición de que el valor esperado de cada variable ambiental según esta distribución coincida con la media empírica de los datos de presencia (Scheldeman y van Zonneveld, 2011; Romo et al., 2012). El MaxEnt genera sus propias ausencias, por lo que no es necesario introducir datos de este tipo en el programa (Phillips et al., 2006). De este modo, el modelo de predicción, se materializa en un mapa que representa las áreas con condiciones climáticas adecuadas o no, para la presencia de la especie (Phillips et al., 2006). El resultado del modelo expresa el valor de idoneidad del hábitat, para la especie como una función de las variables ambientales. Un alto valor de la función de distribución en una celda determinada, indica que ésta presenta condiciones muy favorables para la presencia de la especie.

Se generaron 10 modelos binarios (presencia $=1$, ausencia $=0$ ), por cada especie mediante validación cruzada, la cual permite garantizar la independencia entre estos grupos (Torres y Jayat, 2010). En la validación cruzada se dividen los datos de presencia de las especies en 10 subconjuntos. Uno de los subconjuntos se utiliza para la validación de datos y los nueve restantes para el entrenamiento en el modelado (Cruz et al., 2014). Así mismo, se utilizó un número máximo de 100 iteraciones y el umbral de corte (10 Percentile Training Presence), para maximizar la sensibilidad y minimizar la especificidad (Phillips et al., 2006). Este umbral de corte significa que el $90 \%$ de los puntos de presencia, se encuentran dentro del área potencial, mientras que el $10 \%$ restante de los puntos de presencia, que caen fuera del área potencial corresponden a un ambiente atípico, no incluido dentro de los límites del nicho fundamental (Scheldeman y van Zonneveld, 2011; Valera et al., 2014). Además, con MaxEnt se estima la contribución porcentual de cada variable ambiental a la distribución de la especie.

\section{Evaluación y validación de los modelos}

Para evaluar y validar la capacidad de predicción de los modelos generados por el GARP y el MaxEnt, se utilizó el área bajo la curva o AUC (Area Under the Curve) y el índice de Kappa (Torres y Jayat, 2010; Quintana et al., 2013). El área bajo la curva es la representación gráfica de la capacidad discriminativa de un modelo para todos los posibles puntos de corte y puede interpretarse como la mayor probabilidad de que un punto de presencia seleccionado aleatoriamente, esté ubicado en una celda del raster con un alto valor de probabilidad para la presencia de la especie. Los valores del área bajo la curva se categorizan así: Excelente (AUC $>0,9)$, Buena $(0,8<$ AUC $<0,9)$, Aceptable $(0,7<$ AUC $<0,8)$, Mala $(0,6<$ AUC $<0,7)$ y No Válida $(0,5<$ AUC $<0,6$; Araujo et al., 2005; Elith et al., 2006; Romo et al., 2012; Quintana et al., 2013).

Con el índice de Kappa se mide el desempeño global del modelo. Está definido como la precisión de la predicción de que el modelo se ajusta a la muestra de evaluación, más allá de lo esperado por azar. Los valores de este índice se ubican en el rango 0 - 1; cuanto más cerca esté de 1, mejor será el desempeño del modelo y puede interpretarse según el siguiente baremo: $\mathrm{K}<0,4$, mal desempeño; 0,4 < $\mathrm{K}<0,75$, buen desempeño; y $\mathrm{K}>$ 0,75 , excelente desempeño de los modelos (Naoki et al., 2006; Quintana et al., 2013).

\section{RESULTADOS}

En la Figura 2, se muestran los mapas de distribución potencial generados por el GARP y el MaxEnt, para las principales especies de flebotominos transmisores de Leishmania en el Estado Mérida. En términos generales, ambos métodos centran la distribución de los flebotominos, dentro del Estado Mérida en el Valle del Mocotíes (municipios de Antonio Pinto Salinas, Guaraque, Rivas Dávila, Tovar y Zea), la zona Panamericana (municipios de Alberto Adriani, Caracciolo Parra Olmedo, Julio Cesar Salas, Obispo Ramos de Lora y Tulio Febres Cordero) y los municipios de Andrés Bello, Sucre y Campo Elías. 


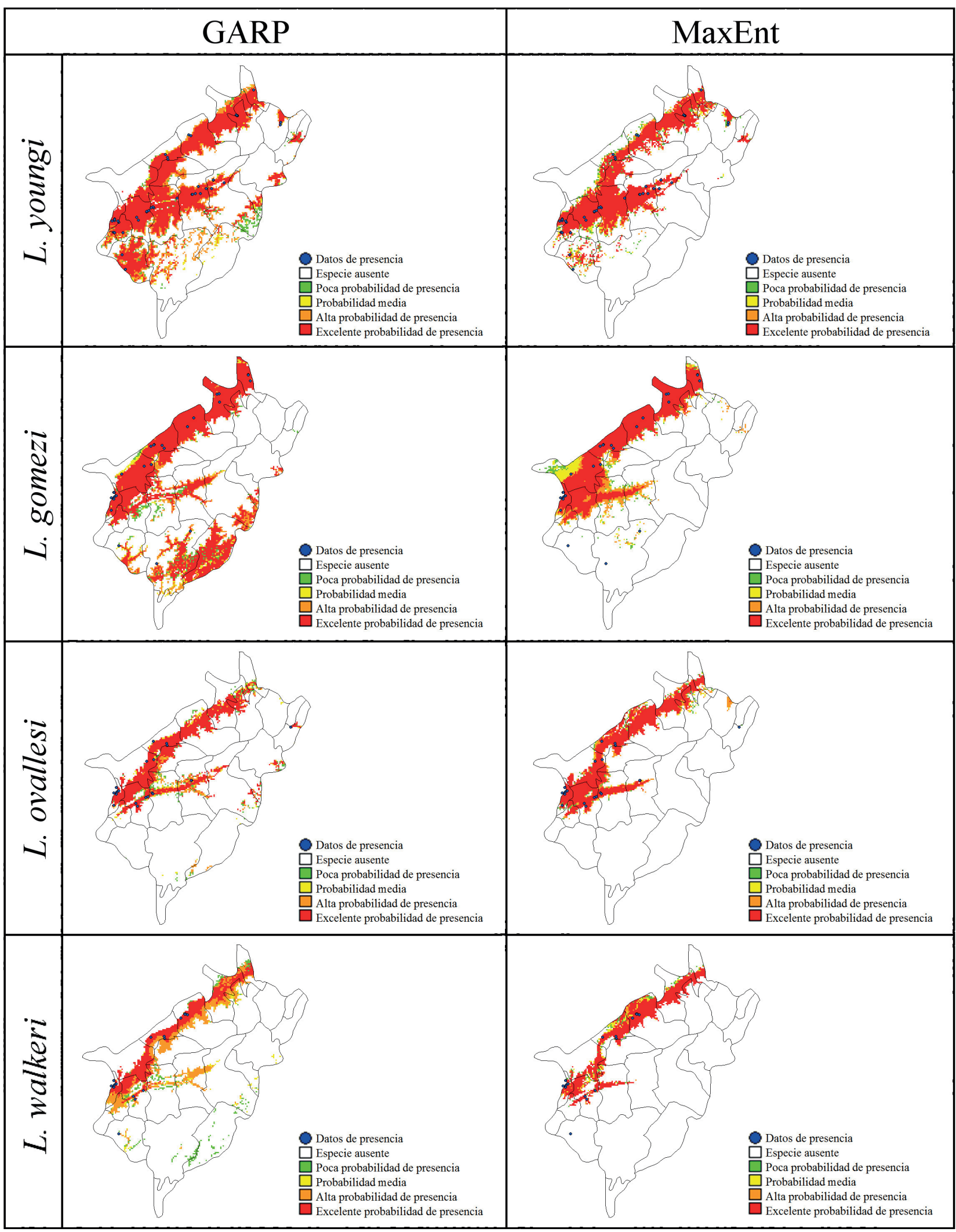

Figura 2. Mapas predictivos de la distribución potencial de L. youngi, L. gomezi, L. ovallesi y L. walkeri en el estado Mérida, Venezuela generados por los modelos GARP y MaxEnt en base en 19 variables bioclimáticas. Los puntos de color azul representan los puntos de presencia de las especies y el color rojo indica las mejores condiciones climáticas para la presencia de las especies en el estado Mérida. 
Los análisis de los modelos de distribución potencial utilizando el GARP y el MaxEnt muestran una distribución potencial diferenciada entre especie; $L$. youngi y $L$. gomezi fueron las especies que presentaron mayor amplitud en su distribución espacial, con más afinidad climática en el área de estudio, mientras que el nicho fundamental delineado para las especies $L$. ovallesi y L. walkeri, es de menor amplitud, con una distribución espacial más restringida, limitada a las zonas del Valle del Mocotíes y la zona Panamericana del Estado Mérida.

Los modelos de distribución potencial mostraron valores del área bajo la curva (AUC), por encima del parámetro de predicción al azar $(0,5)$. El GARP estimó un valor de 0,979 para L. youngi, 0,969 para L. gomezi, 0,999 para $L$. ovallesi y 0,999 para L. walkeri. Por otro lado MaxEnt estimó un valor de 0,995 para L. youngi, 0,990 para $L$. gomezi, 0,997 para L. ovallesi y 0,996 para L. walkeri. Por tanto, ambos modelos presentaron una excelente capacidad de predicción. Así mismo, el estadístico de Kappa indica una buena predicción en ambos modelos, para el GARP se obtuvieron valores de 0,735 para $L$. youngi, 0,627 para L. gomezi, 0,522 para L. ovallesi y 0,509 para $L$. walkeri y con MaxEnt, se mostró un mejor ajuste en la predicción del modelo, excepto para la especie $L$. ovallesi. Los valores fueron de 0,770 para L. youngi, 0,973 para L. gomezi, 0,467 para L. ovallesi y 0,562 para L. walkeri.
En la Tabla 2, se muestra la contribución porcentual de las 19 variables bioclimáticas a cada especie. Esta contribución se generó con el modelo obtenido con MaxEnt. El algoritmo de máxima entropía mostró que la variable más determinante en la distribución espacial de todas las especies de Lutzomyia estudiadas, fue la precipitación en el trimestre más lluvioso (BIO16), con contribuciones entre $37,2 \%$ y 45,3 \%. También se observó que cada especie presenta una caracterización particular de las variables bioclimáticas. Para $L$. youngi la segunda variable bioclimática en orden de importancia, fue la temperatura promedio en el trimestre más caluroso (BIO10), con 20,2\%, seguida por la isotermalidad $\left({ }^{\circ} \mathrm{C}\right)$ (BIO3) con $6,7 \%$ y la estacionalidad de temperatura (\%) (BIO4), con 6,6 \%. Para las especies L. gomezi, L. ovallesi y L. walkeri, la segunda variable en orden de importancia fue la estacionalidad de la precipitación (BIO15), con valores entre 13,4 y 26,4 \% . La tercera variable bioclimática más determinante en la distribución de L. gomezi, fue la estacionalidad de la temperatura (\%) (BIO4), con 7,9\%, seguida de la isotermalidad $\left({ }^{\circ} \mathrm{C}\right)(\mathrm{BIO})$, con el 6,8 \%. Para L. ovallesi, la tercera variable bioclimática más determinante fue la estacionalidad de la temperatura (\%) (BIO4), con el $12,7 \%$, seguida por el rango diurno de temperatura $\left({ }^{\circ} \mathrm{C}\right)$ (BIO2), con el 9,6\%. Y por último para L. walkeri, las variables más relevantes fueron la isotermalidad $\left({ }^{\circ} \mathrm{C}\right)$ (BIO3), con 8,0 \%, seguida por la precipitación en el período más seco (mm) (BIO14), con 8,0 \%.

Tabla 2. Contribución porcentual de cada variable climática a la predicción de la distribución potencial de las especies L. youngi, L. gomezi, L. ovallesi y L. walkeri, mediante el modelo de Máxima Entropía.

\begin{tabular}{|c|c|c|c|c|c|}
\hline & Variables bioclimáticas & L. youngi & L. gomezi & L. ovallesi & L. walkeri \\
\hline $\mathrm{BIO} 1$ & Temperatura promedio anual $\left({ }^{\circ} \mathrm{C}\right)$ & 0 & 0,3 & 0,1 & 0,2 \\
\hline BIO2 & Rango diurno de temperatura $\left({ }^{\circ} \mathrm{C}\right)$ & 1,2 & 5,5 & 9,6 & 6,2 \\
\hline BIO3 & Isotermalidad $\left({ }^{\circ} \mathrm{C}\right)$ & 6,7 & 6,8 & 7,6 & 8,0 \\
\hline BIO4 & Estacionalidad de temperatura (\%) & 6,6 & 7,9 & 12,7 & 7,6 \\
\hline $\mathrm{BIO5}$ & Temperatura máxima del período más caliente $\left({ }^{\circ} \mathrm{C}\right)$ & 4 & 0 & 0 & 0 \\
\hline $\mathrm{BIO6}$ & Temperatura mínima del período más frío $\left({ }^{\circ} \mathrm{C}\right)$ & 0 & 0,1 & 0,4 & 1,5 \\
\hline $\mathrm{BIO} 7$ & Rango anual de temperatura $\left({ }^{\circ} \mathrm{C}\right)$ & 0 & 0 & 0 & 0 \\
\hline $\mathrm{BIO} 8$ & Temperatura media en el trimestre más lluvioso $\left({ }^{\circ} \mathrm{C}\right)$ & 0,2 & 1,4 & 0,4 & 0,3 \\
\hline BIO9 & Temperatura promedio en el trimestre más seco $\left({ }^{\circ} \mathrm{C}\right)$ & 0 & 0 & 0 & 0 \\
\hline BIO10 & Temperatura promedio en el trimestre más caluroso $\left({ }^{\circ} \mathrm{C}\right)$ & 20,2 & 0,8 & 4,5 & 4,5 \\
\hline BIO11 & Temperatura promedio en el trimestre más frío $\left({ }^{\circ} \mathrm{C}\right)$ & 0 & 3,5 & 3,5 & 3,2 \\
\hline BIO12 & Precipitación anual (mm) & 0,2 & 0 & 0 & 0,1 \\
\hline
\end{tabular}


Tabla 2. Continuación.

\begin{tabular}{|c|c|c|c|c|c|}
\hline & Variables bioclimáticas & L. youngi & L. gomezi & L. ovallesi & L. walkeri \\
\hline BIO13 & Precipitación en el período más lluvioso (mm) & 5,9 & 0 & 1,2 & 0 \\
\hline BIO14 & Precipitación en el período más seco (mm) & 4,1 & 5,3 & 4,8 & 8,0 \\
\hline BIO15 & Estacionalidad de la precipitación (\%) & 1,4 & $26,4^{*}$ & 13,4 & 19,6 \\
\hline BIO16 & Precipitación en el trimestre más lluvioso (mm) & $45,3^{*}$ & $37,2^{*}$ & $38,9^{*}$ & $38,5^{*}$ \\
\hline BIO17 & Precipitación en el trimestre más seco (mm) & 0,1 & 0 & 0 & 0 \\
\hline BIO18 & Precipitación en el trimestre más caluroso (mm) & 2,5 & 1,4 & 1,2 & 0,3 \\
\hline BIO19 & Precipitación en el trimestre más frío (mm) & 1,4 & 3,4 & 1,6 & 1,8 \\
\hline
\end{tabular}

*Variables significativas $(\mathrm{p}<0,05)$

\section{DISCUSIÓN}

La distribución geográfica de las especies de flebotominos está correlacionada con factores ecológicos, climáticos y antropogénicos, que han evolucionado a lo largo de los años en un ciclo característico, impactando directamente en la abundancia del vector y en la consecuente transmisión de la leishmaniasis en distintas regiones (Valderrama et al., 2011). Todos los modelos bioclimáticos utilizados para determinar la distribución potencial de las especies, involucran errores de comisión (clasificar una ausencia como presencia) y de omisión (clasificar una presencia como ausencia) (Peterson y Vieglais, 2001; Anderson et al., 2003). Sin embargo, son métodos formales, rigurosos y válidos, para predecir en un área particular donde pudiera existir una especie de interés. Los modelos de nicho fundamental ayudan a determinar dónde se encuentran las condiciones ambientales más adecuadas para que la especie prospere, en función de parámetros obtenidos de recolectas previas (Romo et al., 2012; Acosta y Vergara, 2013; Valera et al., 2014). Los mapas obtenidos en este trabajo, con ambos modelos, muestran una buena predicción de la distribución potencial de las principales especies de Lutzomyia en el Estado Mérida y son la primera aproximación regional para identificar y caracterizar las zonas climáticamente favorables, en las que estas especies podrían ocurrir de manera natural.

En general, los modelos de distribución geográfica potencial de las especies L. youngi, L. gomezi, $L$. ovallesi y L. walkeri, evidenciaron un buen desempeño tanto con el GARP, como con el MaxEnt y sus rangos de distribución son consistentes entre sí, de acuerdo a la cantidad de registros de presencia de las especies estudiadas. Los resultados sugieren una excelente predicción de los patrones de distribución geográfica de los flebotominos en el área de estudio por ambos métodos, como lo sugiere Guisan y Thuiller (2005). Con ambos métodos de modelización, los valores de AUC se encuentran por encima de 0,9 , lo cual indica que la distribución actual en los hábitats estudiados, se encuentra bien descrita por el clima. Los valores del índice Kappa (por encima de 0,5), indican una buena capacidad predictiva de los modelos. Los dos modelos analizados presentan áreas comunes y muestran la distribución espacial de las especies de flebotominos estudiados en función de las condiciones climáticas del área de estudio.

La distribución poblacional de los flebotominos está controlada por factores endógenos (biológicos) y exógenos (bioclimáticos) y la contribución de cada variable puede afectar de manera particular a cada especie (Yang et al., 2008). Los modelos obtenidos mediante el GARP y el MaxEnt, indican que las áreas desfavorables para la presencia de las especies de flebotominos coinciden con zonas de mayor altura ( $>3000 \mathrm{~m}$ ), que son zonas de vidas con bioclimas poco adecuados, con temperaturas menores de 10 ${ }^{\circ} \mathrm{C}$, las cuales son condiciones inadecuadas para la sobrevivencia de los flebotominos (Ataroff y Sarmiento, 2004; Feliciangeli, 2006; Yarbuth, 2011). En Mérida las zonas actuales y potenciales de distribución de los flebotominos y el riesgo de transmisión de leishmaniasis, están limitados por la presencia de ambientes de páramo con poca vegetación y condiciones climáticas desfavorables. 
Los resultados también mostraron diferencias entre las predicciones de la distribución potencial entre los dos métodos. El GARP arrojó mapas con mayores proporciones de zonas dónde se encuentran las condiciones ambientales más adecuadas para L. youngi y L. gomezi, con respecto al MaxEnt. El GARP predijo una mayor distribución potencial de L. youngi, la cual podría estar presente en el municipio Guaraque, mientras que para L. gomezi predice una amplia distribución potencial, que abarca los municipios Guaraque, Arzobispo Chacón, Aricagua y parte del Libertador. Inclusive, aunque en menor proporción, el GARP proporcionó un mejor ajuste en la predicción para las especies L. ovallesi y L. walkeri, con relación a lo encontrado con MaxEnt para estas especies. Este hecho sugiere que el modelaje con GARP presenta una fuerte capacidad predictiva, como lo informan otros autores (Naoki et al., 2006; Terribile et al., 2010; Wang et al., 2010; Pliscoff y Fuentes, 2011).

Aunque el modelo de distribución potencial de máxima entropía aporta un menor ajuste predictivo para las especies en estudio, este método de predicción presenta una relación más adecuada con las variables bioclimáticas. El MaxEnt se ha usado ampliamente, en los últimos años por diferentes autores en virtud de la robustez de sus predicciones (Fischer et al., 2011; González et al., 2011; Pliscoff y Fuentes, 2011). El modelo de MaxEnt dio una mejor idea general del nicho fundamental de las principales especies de Lutzomyia. El algoritmo de máxima entropía mostró que la variable climática "precipitación en el trimestre más lluvioso" (BIO16) fue la de mayor influencia en la distribución potencial de estas especies de Lutzomyia.

El riesgo de transmisión de leishmaniasis se incrementa en las zonas de superposición de varias especies transmisoras en espacio y tiempo. Con la modelización con MaxEnt, se identificó esas áreas de interacción entre las especies L. youngi, L. gomezi, L. ovallesi y L. walkeri, lo que permitió relacionarla con la variable climática del trimestre más lluvioso (BIO16). Lo anterior profundiza el conocimiento sobre los patrones actuales y potenciales de ocurrencia de estas especies en el Estado Mérida, con relación a las variables climáticas que las caracterizan, considerándose el modelaje con el MaxEnt como una herramienta poderosa para la prevención de la leishmaniasis. La variabilidad del clima del Estado Mérida muestra un régimen bimodal anual, con períodos de alta precipitación de abril a junio y de agosto a octubre, y un período de baja precipitación de enero a marzo (Ataroff y Monasterio, 1987; Sáez y Martelo, 2007), lo que altera la disponibilidad de la materia orgánica utilizada para la deposición de los huevos y alimento de las larvas (Young y Duncan, 1994; OMS, 2010), situación que afecta el ciclo biológico de los flebotominos.

El mayor número de ocurrencias de casos de leishmaniasis se presenta durante los primeros meses del año (época seca), luego van descendiendo hasta alcanzar su mínimo en la época de lluvia (De Lima et al., 2010). Esto explica el hecho de que la distribución de los flebotominos esté determinada por los "meses con mayor precipitación", variable bioclimática que ejerce la mayor influencia en la dinámica poblacional de estas especies, ya que durante su vigencia se crean ambientes húmedos que favorecen la postura de los huevos en sitios adecuados para su posterior crecimiento y el desarrollo de los estadios larvales.

La temperatura promedio del trimestre más caluroso (BIO10), fue la segunda variable bioclimática más influyente en la distribución potencial de L. youngi, seguida por la isotermalidad (BIO3) y la estacionalidad de temperatura (BIO4). El comportamiento de estas variables permite una mayor distribución potencial de L. youngi, en comparación con las otras especies. En efecto, aunque $L$. youngi juega un papel relevante en la transmisión de leishmaniasis, en zonas altas (8001200 m) del valle del Mocotíes (Añez et al., 1998; Nieves et al,. 2008; Rondón, 2015), el MaxEnt indicó que su distribución geográfica podría abarcar también zonas bajas, por ejemplo el municipio Caracciolo Parra Olmedo, a una altura de $159 \mathrm{~m}$, e incluso en zonas más altas, por ejemplo el municipio Libertador, a una altura de 2099 m, lo que podría incrementar el riesgo de transmisión de la enfermedad. Estos resultados coinciden con otros estudios en donde se ha informado la predominancia de L. youngi, en zonas localizadas a lo largo de un gradiente altitudinal entre los 50 y 1800 m (Añez et al., 1994; Rondón, 2015).

Recientemente, Rondón (2015), afirmó que L. gomezi es la especie predominante en los municipios de menor altura y mayor temperatura, de la zona Panamericana del Estado Mérida. El modelo MaxEnt de distribución espacial de L. gomezi sugiere también una posible expansión de su distribución potencial a una altura de 1664 m (Municipio Guaraque). El incremento en la distribución espacial de L. gomezi en el Estado Mérida es una clara evidencia de que esta especie ha sabido aprovechar los factores abióticos, así como cambios ecológicos de origen antrópico (Nieves et al., 2014a). 
L. gomezi está reconocida como uno de los vectores más importantes de leishmaniasis en diferentes focos del piedemonte andino ( $<400 \mathrm{~m}$ ) (Añez et al., 1998; Nieves et al., 2008; Nieves et al., 2014b; Rondón, 2015). Por tanto, existe el riesgo de que esta especie amplíe su distribución geográfica, lo que incrementaría el riesgo de transmisión de leishmaniasis. Las diferencias entre la distribución potencial modelada con GARP y MaxEnt para L. gomezi, podría deberse a que las variables bioclimáticas que la determinan son principalmente la estacionalidad de la precipitación (BIO15) y en menor grado, la temperatura en el trimestre más lluvioso (BIO8) y la precipitación en el trimestre más frio (BIO19), mientras que la temperatura del trimestre más caluroso (BIO10), no resultó determinante, lo que sugiere que la proyección con el MaxEnt da una distribución más ajustada con L. gomezi, en contraste con lo ocurrido en las demás especies.

Por otro lado, Feliciangeli y Rabinovich (1998), encontraron que la mayor abundancia de L. ovallesi estuvo relacionada con las variables climáticas menor temperatura y precipitación. Esta especie está identificada como transmisora de Leishmania braziliensis y Leishmania mexicana, en ambientes boscosos (Añez et al., 1994; Jorquera et al., 2005; Rondón, 2015). Para las especies L. ovallesi y L. walkeri, los modelos mostraron una distribución potencial restringida. Según el MaxEnt, la variable bioclimática que determinó la distribución de L. ovallesi fue el rango diurno de temperatura (BIO2), en tanto que la distribución de L. walkeri estuvo mayormente condicionada por la precipitación en el período más seco (BIO14). Esto explica que L. ovallesi se haya detectado en zonas medias (400-800 m) y que su distribución potencial abarque una zona situada a los $1811 \mathrm{~m}$, en el Municipio Cardenal Quintero. De igual manera para $L$. walkeri, se determinó un rango de distribución potencial en zonas de $1600 \mathrm{~m}$ en el Municipio Guaraque y esta especie ha registrado con abundancia bajas en las zonas medias del Estado (400 y 800 m) (Nieves et al., 2014b).

La fiabilidad de estos modelos puede verse afectada por el sesgo espacial de los datos de presencia, lo que debe tomarse en cuenta, al interpretar los modelos resultantes. Estos sesgos pueden englobarse a grandes rasgos en dos categorías: los que provienen de muestreos por rutas aleatorias y los que se derivan de zonas insuficientemente muestreadas por la complejidad del acceso, debido a carencia o mal estado de las vías de comunicación. Sin embargo, la importancia de los modelos no debe subestimarse, ya que proporcionan un punto de partida útil para predecir la distribución potencial de las especies a diferentes escalas y regiones (Peterson y Vieglais, 2001; Anderson et al., 2003).

\section{CONCLUSIONES}

Los resultados muestran que las técnicas GARP y MaxEnt generan predicciones de alta precisión, en lo que respecta a la distribución potencial de las principales especies de flebotominos en el Estado Mérida. Ambas técnicas de modelización comparten un patrón similar y se ajustan al rango de distribución actual de las especies. El modelaje con GARP sugiere una mayor amplitud en las áreas de distribución potencial de las especies L. youngi y L. gomezi, lo que demuestra la eficacia del modelo y proporcionando una información valiosa sobre la dispersión de las especies. Sin embargo, el modelaje con MaxEnt genera predicciones más ajustadas de probabilidades de ocurrencia de las especies de flebotominos, incluso determina las variables bioclimáticas que caracterizan la distribución potencial de cada especie. Finalmente, se aportan modelos predictivos de distribución potencial de los flebotominos en el Estado Mérida, que pueden ser usados para determinar las áreas de posible presencia e identificar zonas de riesgo de transmisión, como una herramienta en la prevención y control de la leishmaniasis. Se recomienda el uso del MaxEnt como técnica de modelización para entender la dinámica de distribución de Lutzomyia, ya que presenta un mejor desempeño con relación a las variables bioclimáticas que determinan la distribución potencial de cada especie.

\section{AGRADECIMIENTOS}

Este trabajo fue realizado en el Estado Mérida, gracias a la colaboración de todas aquellas familias que con su amabilidad apoyaron la realización de las capturas peridomiciliarias de los flebotominos, a la Universidad de Los Andes y al apoyo financiero del Proyecto Estratégico-FONACIT (Fondo Nacional de Ciencias, Tecnología e Innovación) No 2011000371, bajo la Coordinación de la Dra. Elsa Nieves.

\section{BIBLIOGRAFÍA}

Abdel, M., Annajar, B., Hanafi, H., Obenauer, P. 2012. The Potential Distribution of Phlebotomus papatasi (Diptera: Psychodidae) in Libya Based on Ecological Niche Model. Journal of Medical Entomology. 49(3):739-745. 
Acosta, L. y Vergara, L. 2013. New records and distribution modeling of Gryneorensis (Sørensen) (Opiliones: Cosmetidae) support the Mesopotamian-Yungas disjunction in subtropical Argentina. Zootaxa 3736(2):143-158.

Alvar, J., Vélez, I.D., Bern, C., Herrero, M., Desjeux, P., Cano, J., Jannin, J., den Boer, M., 2012. WHO Leishmaniasis control team. Leishmaniasis worldwide and global estimates of its incidence. PLOS ONE 7, e35671.

Anderson, R., Lew, D., Peterson, A. 2003. Evaluating predictive models of species' distributions: criteria for selecting optimal models. Ecological Modelling 162:211-232.

Añez, N., Lugo, A., Loaiza, A., Nieves, E., Orozco, J. 1994. Sugar in the alimentary canal of Lutzomyia youngi (Diptera: Phlebotominae). Medical and Veterinary Entomology 8: 38-42.

Añez, N., Cazorla, D., Nieves, E., Chataing, M., De Yarbuh, A. 1998. Epidemiologia de la Leishmaniasis tegumentaria en Mérida, Venezuela. Diversidad y dispersión de especies flebotominas en tres pisos altitudinales y su posible rol en la trasmisión de la enfermedad. Memórias do Instituto Oswaldo Cruz 83(4):455-463.

Araujo, M., Pearson, R., Thuiller, W. y Erhard, M. 2005. Validation of species climate impact models under climate change. Global Change Biology 11(9):1504-1513.

Ataroff, M. y Monasterio, M. 1987. Ecología y desarrollo en Los Andes tropicales: pisos de vegetación y asentamientos humanos. En: Simposio Ecología de Tierras Altas. IV Congreso Latinoamericano de Botánica 1:65-81.

Ataroff, M. y Sarmiento, L. 2004. Las unidades ecológicas de los Andes de Venezuela. En: La Marca, E., Soriano, P. (eds). Reptiles de Los Andes de Venezuela. Fundación Polar, Codepre-ULA, Fundacite-Mérida. Biogeos 9-26.

Carnaval, A. y Moritz, C. 2008. Historical climate modelling predicts patterns of current biodiversity in the Brazilian Atlantic forest. Journal of Biogeography 35:1187-1201.

Chapman, A.D. y Busby, J.R.1994. Linking plant species information to continental biodiversity inventory, climate modeling and environmental monitoring. In: Miller, R.I. (ed.). Mapping the diversity of nature. Chapman y Hall, 48 London.

Chen, X., Lei, Y. 2012. Effects of Sample Size on Accuracy and Stability of Species Distribution Models: A Comparison of GARP and MaxEnt. Recent Advances in Computer Science and Information Engineering 125:601-609.
Cruz, G., Villaseñor, J., López, L., Martínez, E., Ortiz, E. 2014. Selección de predictores ambientales para el modelado de la distribución de especies en MaxEnt. Revista Chapingo 20(2):187-201.

De Lima, H., Carrero, J., Rodríguez, A., De Guglielmo, S., Rodríguez, R. 2006. Trypanosomatidae de importancia en salud pública en animales silvestres y sinantrópicos en un área rural del municipio Tovar del estado Mérida, Venezuela. Biomédica 26:42-50.

De Lima, H., Borges, R., Escobar, J., Convit, J. 2010. Leishmaniasis Cutánea Americana en Venezuela: Un análisis clínico epidemiológico a nivel nacional y por entidad federal, 1988-2007. Boletín de Malariología y Salud Ambiental 50: 2.

Donalisio, M., Peterson, A., Costa, P., da Silva, F., Valencia, H., Shaw, J., Filho, S. 2012. Microspatial Distributional Patterns of Vectors of Cutaneous Leishmaniasis in Pernambuco, Northeastern Brazil. Journal of Tropical Medicine Article ID 642910.1-8.

Elith, J., Graham, C., Anderson, R., Dudik, M., Ferrier, S. 2006. Novel methods improve prediction of species' distributions from occurrence data. Ecography 29:129-151.

Feliciangeli, D. 1988. La fauna flebotómica (Díptera, Psychodidae) en Venezuela: 1 Taxonomía y distribución geográfica. Boletín de la Dirección de Malariología y Saneamiento Ambiental 28(3-4):1-15.

Feliciangeli, D., Rabinovich, J. 1998. Abundance of Lutzomyia ovallesi but not Lu. gomezi correlated with cutaneous leishmaniasis incidence in north-central Venezuela. Medical and Veterinary Entomology 12: 121-131.

Feliciangeli, D. 2006. Sobre los flebótomos (Diptera: Psychodidae: Phlebotominae), con especial referencia a las especies conocidas en Venezuela. Acta Biologia Venezolana 26(2): 61-80.

Fischer, D., Thomas, S., Beierkuhnlein, C. 2011. Modelling climatic suitability and dispersal for disease vectors: the example of a phlebotomine sandfly in Europe. Elsevier. 7:164-169.

Foley, D., Klein, T., Kim, H., Brown, T., Wilkerson, R., Rueda, L. 2010. Validation of ecological niche models for potential malaria vectors in the Republic of Korea. Journal of the American Mosquito Control Association 26(2):210-213. 
Githeko, A., Lindsay, S., Confalonieri, U., Patz, J. 2009. El cambio climático y las enfermedades transmitidas por vectores: un análisis regional. Revista Virtual Redesma 3(3):1-18.

González, C., Rebollar, E., Ibáñez, S., Becker, I., Martínez, E., Peterson, A., Sánchez, V. 2011. Current Knowledge of Leishmania Vectors in Mexico: How Geographic Distributions of Species Relate to Transmission Areas. American Journal of Tropical Medicine and Hygiene 85(5):839-846.

Guisan, A., Thuiller, W. 2005. Predicting species distribution: Offering more than simple habitat models. Ecology Letters 8: 993-1009.

Hernández, P., Graham, C., Master, L., Albert, D. 2006. The effect of sample size and species characteristics on performance of different species distribution modeling methods. Ecography 29:773-785.

Hijmans, R., Cameron, S., Parra, J., Jones, P., Jarvis, A. 2005a.Very high-resolution interpolated climate surfaces for global land areas. International Journal of Climatology 25:1965-1978.

Hijmans, R., Guarino, L., Jarvis, A., O’Brien, R., Mathur, P. 2005b. DIVA-GIS, versión 7.5 http://www.diva-gis.org/.

Jorquera, A., González, R., Marchan-Marcano, E., Oviedo, M., Matos, M. 2005. Multiplex-PCR for detection of natural Leishmania infection in Lutzomyia spp. captured in an endemic region for cutaneous Leishmaniasis in state of Sucre, Venezuela. Memorias del Instituto Oswaldo Cruz 100: 43-46.

Mateo, R., Felicísimo, A., Muñoz, J. 2011. Modelos de distribución de especies: Una revisión sintética. Revista Chilena de Historia Natural. 84:217-240.

Naoki, K., Gómez, M., López, R., Meneses, R., Vargas, J. 2006. Comparación de modelos de distribución de especies para predecir la distribución potencial de vida silvestre en Bolivia. Ecología en Bolivia 41(1): 65-78.

Nieves, E., Villarreal, N., Rondón, M., Sánchez, M., Carrero, J. 2008. Evaluación de conocimientos y prácticas sobre la Leishmaniasis tegumentaria en un área endémica de Venezuela. Biomédica 28:347-56.

Nieves, E., Oraá, L., Rondón, Y., Sánchez, M., Sánchez, Y., Rojas, M., Rondón, M., Rujano, M., González, N., Cazorla, D. 2014a. Effect of Environmental Disturbance on the Population of Sandflies and Leishmania Transmission in an Endemic Area of Venezuela. Journal of Tropical Medicine 2014:1-7.

Nieves, E., Oraá, L., Rondón, Y., Sánchez, M., Sánchez, Y., Rujano, M., Rondón, M., Rojas, M., González, N., Cazorla, D. 2014b. Riesgo de transmisión de Leishmania (Kinetoplastida: Trypanosomatidae) en Mérida Venezuela. Avances en Biomedicina 3: 57-64.

OMS. 2010. Control de la Leishmaniasis. Serie de Informes Técnicos 949. Ginebra, Suiza.

OMS. 2015. Leishmaniasis- Nota descriptiva $N^{\circ} 375$ http://www.who.int/mediacentre/factsheets/fs375/es/. Consultado: 9 de octubre de 2014.

Pearson, R., Thuiller, W., Araújo, M., Martinez, E., Brotons, L., McClean, C., Miles, L., Segurado, P., Dawson, T., Lees, D. 2006. Model-based uncertainty in species range prediction. Journal of Biogeography 33:1704-1711.

Peterson, A., Vieglais, D. 2001. Predicting Species Invasions Using Ecological Niche Modeling: New Approaches from Bioinformatics Attack a Pressing Problem. BioScience 51(5):363-371.

Peterson, A., Papes, M., Eaton, M. 2007. Transferability and model evaluation in ecological niche modeling: a comparison of GARP and MaxEnt. Ecography 30: 550-560.

Phillips, J., Anderson, P., Schapire, E. 2006. Maximum entropy modeling of species geographic distributions. Ecological Modeling. 190:231-259.

Phillips, J., Dudik, M. 2008. Modeling of species distributions with MaxEnt: new extensions and a comprehensive evaluation. Ecography 31:161-175.

Pliscoff, P., Fuentes, T. 2011. Modelación de la distribución de especies y ecosistemas en el tiempo y en el espacio: una revisión de las nuevas herramientas y enfoques disponibles. Revista de Geografía Norte Grande 48:61-79.

Quintana, M., Salomón, O. 2011. Lutzomyia neivai, vector of Leishmaniasis in north western Argentina: preliminarydistributionmap. Acta Biológica Venezuelica $31(2): 33-36$.

Quintana, M., Salomón, O., Guerra, R., Lizarralde, M., Fuenzalida, A. 2013. Phlebotominae of epidemiological importance in cutaneous Leishmaniasis in north western Argentina: risk maps and ecological niche models. Medical and Veterinary Entomology 27:39-48. 
Rodríguez, N., Carrero, R., De Lima, H., Sandoval, I., Fernández, A., Barrios, M. 2007. Impacto de Fenómenos Naturales (Deslaves y vaguadas) sobre la epidemiologia de la Leishmaniasis cutánea en zonas del estado Mérida. Salus 11:43-47.

Romo, H., Sanabria, P., García, E. 2012. Predicción de los impactos del cambio climático en la distribución de lepidópteros del género Boloria Moore, 1900 en la Península Ibérica (Lepidoptera: Nymphalidae). SHILAP Revista de Lepidopterología 40(158):1-20.

Rondón, Y. 2015. Detección Parasitológica y Molecular de Leishmania en el Intestino de Flebotominos Vectores. Tesis. Universidad de Los Andes, Mérida, Venezuela.

Rubio, G. y Acosta, L. 2011. Geographical distribution of the space-weaving spider, Chibcheasalta, from northwestern Argentina: New records and bioclimatic modeling. Journal of Insect Science. 11(54):1-14.

Sáez, V., Martelo, M. 2007. Posibles cambios geográficos para la expansión de enfermedades metaxénicas en la región centro-norte de Venezuela. Revista Geográfica Venezolana 48(1):83-99.

Sánchez, I., Liria, J., Feliciangeli, M. 2015. Ecological Niche Modeling of Seventeen Sandflies Species (Diptera, Psychodidae, Phlebotominae) from Venezuela. International Journal of Zoology 2015:1-10.

Scachetti, R. 2002. Desktop Garp: a software package for biodiversity and ecologic research. United States: The University of Kansas Biodiversity Research Center. http:// www.nhm.ku.edu/desktopgarp/.

Scheldeman, X., van Zonneveld, M. 2011. Manual de capacitación en análisis espacial de diversidad y distribución de plantas. Bioversity International. 1-186.

Stockdale, L., Newton, R. 2013. A Review of Preventative Methods against Human Leishmaniasis Infection. PLoS Neglected Tropical Diseases 7(6):1-15.

Stockwell, D., Noble, I. 1992. Induction of set of rules from animal distribution data: a robust and informative method of analysis. Mathematics and Computers in Simulation 33: 385-390.

50

Stockwell, D., Peters, D. 1999.The GARPmodelling system: problems and solutions to automated spatial prediction. International Journal of Geographical Information Science 13(2):143-158.
Terribile, L., Diniz, J., De Marco, P. 2010. How many studies are necessary to compare nichebased models for geographic distributions? Inductive reasoning may fail at the end. Brazilian Journal of Medical and Biological Research 70(2): 263-269.

Tognelli, M., Roig, S., Marvaldi, A., Flores, G., Lobo, J. 2009. An evaluation of methods for modelling distribution of Patagonian insects. Revista Chilena de Historia Natural 82:347-360.

Torres, R., Jayat, P. 2010. Modelos predictivos de distribución para cuatro especies de mamíferos (cingulata, artiodactyla y rodentia) típicas del Chaco en Argentina. Mastozoología Neotropical 17(2):335-352.

Traviezo, L. 2007. El uso del sistema de información geográfico (SIG), en el estudio de la Leishmaniasis cutánea en el municipio Andrés Eloy Blanco, Estado Lara, Venezuela. Boletín Médico de Postgrado Universidad Centroccidental Lisandro Alvarado. 23:1-4.

Thomson, M.C., Elnaiem, D.A., Ashford, R.W. y Connor, S.J. 1999. Towards a kala azar risk map for Sudan: mapping the potential distribution of Phlebotomus orientalis using digital data of environmental variables. Tropical Medicine International Health 4(2):105-13

Valderrama, A., Tavares, M., Andrade, J. 2011. Anthropogenic influence on the distribution, abundance and diversity of sandfly species (Diptera: Phlebotominae: Psychodidae), vectors of cutaneous Leishmaniasis in Panama. Memórias do Instituto Oswaldo Cruz 106(8):1024-1031.

Varela, S., Mateo, R., García, R., Fernández, F. 2014. Macroecología y ecoinformática: sesgos, errores y predicciones en el modelado de distribuciones. Ecosistemas 23(1):46-53.

Vessella, F., Schirone, B. 2013. Predicting potential distribution of Quercussuber in Italy based on ecological niche models: Conservation insights and reforestation involvements. Forest Ecology and Management 304:150-161.

Wang, X., Huang, X., Jiang, L., Qiao, G. 2010. Predicting potential distribution of chestnut phylloxerid (Hemiptera: Phylloxeridae) based on GARP and Maxent ecological niche models. Journal of Applied Entomology 134: 45-54.

Wisz, M., Hijmans, R., Li, J., Peterson, A., Graham, C., Guisan, A. 2008.Predicting Species Distributions Working Group. Effects of sample size on the performance of species distribution models. Diversity and Distributions 14:763-773. 
Yang, G., Brook, B., Whelan, P., Cleland, S., Bradshaw, C. 2008. Endogenous and exogenous factors controlling temporal abundance patterns of tropical mosquitoes. Ecological Applications 18(8):2028-2040.

Yarbuh, U. 2011. Aspectos Socio-Ambientales de la Leishmaniasis Cutánea en el Estado Mérida. Tesis de Maestría. Centro Interamericano del Desarrollo e
Investigación Ambiental y Territorial. Universidad de Los Andes, Mérida, Venezuela.

Young, D. y Duncan, M. 1994. Guide to the identification and geographic distribution of Lutzomyia sandflies in México, the West Indies, Central and South America (Diptera: Psychodidae). Memoris of the American Entomological 54:779-881.

Fecha de recepción: 27/08/2015

Fecha de aceptación: 12/11/2015

Para citar este artículo: Rujano, M., L. Oraá, Y. Rondón, M. Sánchez, M. Rondón, Y. Sánchez, M. Rojas, N. González, D Cazorla y E. Nieves. 2015. Modelo de la distribución de Lutzomyia (Diptera: Psychodidae) vectores de leishmaniasis en Mérida Venezuela. Revista Intropica Vol. 10: 37 - 51 